\title{
Publisher Correction: Navigating for reward
}

Marielena Sosa (1) and Lisa M. Giocomo (1)

Correction to: Nature Reviews Neuroscience https://doi.org/10.1038/s41583-021-00479-z, published online 06 July 2021.

In Fig. 2d of this article; the text below the arrow in the right hand panel should have read "Place fields shift towards reward". The error has now been corrected in the HTML and PDF versions of the article.

https://doi.org/10.1038/s41583-021-00505-0 I Published online 23 July 2021

๑) Springer Nature Limited 2021 